\title{
Carcinoma In Situ
}

\section{DIAGNOSTICO Y MANEJO}

\section{BIOPSIA FRENTE A CONO}

\section{Hospital de San Juan de Dios de Cali 1970 - 1976 \\ DR.: NESTOR H. AMOROCHO F. \\ DR.: FABIO HENAO A.}

\section{INTRODUCCION}

El cáncer de cuello uterino ocupa el primer lugar entre las neoplasias que afectan a la población Colombiana. (3).

Corresponde a la mitad de los cánceres que afectan el sistema reproductivo femenino, (3) (1) incluyendo la mama y una tercera parte de los tumores que se presentan en mujeres en la ciudad de de Cali (5).

La introducción de la citología marca, en la historia de la práctica. ginecológica, un cambio significativo en el diagnóstico del cáncer invasor de cuello uterino, permitiendo encontrarlo en etapa más temprana y sirviendo de guía hacia el diagnóstco de sus precursores: la displasia y el carcinoma in situ (11) (10) (12). El diagnóstico definitivo de cancer, dado por el estudio histopatológico de especimen de cervix, obtenido por biopsia, fue mejorado notoriamente cuando en 1929 Schiller aporta el método de toma de biopsia previa "aplicación de yodo y raspado del epitelio de la porción vaginal del cuello".

Fue necesario que transcurrieran años, para reconocer universalmente, que tanto la citología como la colposcopia, ideada por Hinselman desde 1925, tenían posibilidades pero tamkién limitaciones en el diagnóstico del cancer de cervix y que ambos métodos se complementan. Dada la falta de suficientes colposcopistas con experiencia es dífícil recurrir a ambos métodos para complementarlos.

En la actualidad, la función principal de la colposcopia consiste en la valoración clínica de pacientes con citología exfoliativa anormal y gran ayuda en la toma de biopsia "dirigida bajo colposcopia" (11) (10).

Se ha invocado la conización del cervix como único procedimiento 
de seguridad diagnóstica histológica, para demostrar la neoplasia (7) $\mathrm{y}$ de otro lado hay quienes, en forma clásica, preconizan efectuar la biopsia como un método inicial de selección, seguida de conización, en casos escogidos (9-2).

Utilizando la prueba de Schiller, para efectuar "biopsia dirigida" del cuello uterino se ha señalado una exactitud de 84 a $97 \%$ para los casos de carcinoma in situ y temprana invasión del estroma (4) (8) (12).

En el presente trabajo presentamos nuestra experiencia en el diagnóstico y manejo del carcinoma in situ de Cervix, a partir de la citología vaginal, para continuar la secuencia biopsia cirugía (histerecmía) obviando el cono, (salvo casos especiales) en busca de una metodología racional que ofrezca un margen de seguridad adecuada, que disminuya labor de histopatología y abarate costos en el diagnóstico y tratamiento de pacientes de este tipo, en el precario medio hospitalario de nuestro país.

\section{MATERIAL Y METODOS}

El estudio se hizo en base al diagnóstico de egreso de pacientes del Servicio de Ginecología del Hospital de San Juan de Dios de Cali, en un lapso de 7 años comprendido entre Enero $1^{\circ}$ de 1970 y Diciembre 31 de 1976.

Es pertinente anotar que nuestro Hozpital durante el periodo anotado y hasta la actualidad, se encuentra en proceso de remodelación y las dificultades locativas inheren. tes a la construcción, se ref́lejan en disminución de disponibilidades en planta física y por ende en reducción de número de camas.

Se tomaron 119 historias clínicas que correspondieron al total de egresos de pacientes con diagnós tico de Carcinoma in situ de cuello uterino, en el lapso anotado.

A todas las pacientes se les había efectuado citología vaginal, rutina establecida en el Servicio de Gine. cología del Hosipital, previa a la -nn-ulta médica en consulta CXterna.

Siguiendo las normas para el manejo de pacientes, se efectúa biopsia de cervix bajo Schiller a todas aquellas cuya citología sea informada grado III o mayor y se hace la valoración clínica respecti. va en base al diagnóstico Histopatológico de la biopsia que confirma el Carcinoma.

Los estados II y subsiguientes que en los primeros años (1970 1973) se diagnosticaron, fueron hospitalizados para complementar su estudio y posterior remisión a otro centro hospitalario para Cobaltoterapia; en la actualidad es. tas pacientes no se hospitalizan. Aquellos casos diagnostidos como $\mathrm{C}^{\prime}$ a. in situ, siguiendo la conducta ya definida desde 1968, en el Servicio de Ginecología del Hospital de San. Juan de Dios de Cali (12), se llevan a Histerectomía, sin efectuar conización previa a la intervención.

Se reserva el Cono sólo para pacientes que no aceptan por razones 
do edad y con miras a conservar la función Obstétrica, la extirpa.ción del útero. En la revisión para este trabajo se encontraron cuatro pacientes a las cuales se les efectuó conización.

RESULTADOS
Este cuadro muestra el total de egresos del Hospital, distribuiido por año y sexo, observándose que la demanda de servicios hospitalarios es mayor para mujeres $58.32 \%$ - que para hombres $41.68 \%$ en el total de los 7 años de la. revisión.

\section{EGRESOS HOSPITALARIOS}

DISTRIBUCION PORCENTUAL POR SEXO Y AÑO

\begin{tabular}{|c|c|c|c|c|c|}
\hline AÑO & MASCULINO & & FEMENINO & & TOTAL \\
\hline & $\mathrm{N}^{\circ}$ & $\%$ & $\mathrm{~N}^{\circ}$ & $\%$ & \\
\hline 1970 & 2659 & 43.84 & 3.406 & 56.16 & .6 .065 \\
\hline 1971 & 2526 & 43.64 & 3.262 & 56.36 & 5.788 \\
\hline 1972 & 2027 & 41.25 & 2.887 & 58.75 & 4.914 \\
\hline 1973 & 2045 & 40.53 & 3.001 & 59.47 & 5.046 \\
\hline 1974 & 1747 & 38.27 & 2.818 & 61.73 & 4.565 \\
\hline 1975 & 1726 & 42.22 & 2.362 & 57.78 & 4.088 \\
\hline 1976 & 1359 & 40.76 & 1.975 & 59.24 & 3.334 \\
\hline TOTAL & 14.089 & 41.68 & 19.711 & 58.32 & 33.800 \\
\hline
\end{tabular}

CUADRO N: 1

DIAGNOSTICO CARCINOMA DE CERVIX (0-IV)

POR EGRESOS FEMENINOS

\begin{tabular}{cccc}
\hline \multicolumn{2}{c}{ EGRESOS FEMENINOS } & Ca. CERVIX \\
$\mathrm{N}^{\circ}$ & $\%$ & $\mathrm{~N}^{\circ}$ & $\%$ \\
\hline 19.711 & 58.32 & 229 & 1.16 \\
\hline
\end{tabular}

\section{CUADRO № 2}

Efectuado el análisis estadístico de los diagnósticos que correspondieron a la población femenina atendida en el periodo 1970-1976, se er. contró que el $1.16 \%$ fué Carcinoma de Cervix, sin hacer consideración del estadío clínico. 
Dirigiendo la investigación diag:nóstica hacia el Carcinoma in situ. de Cervix y efectuado el cerrimiento entre el número global de pacientes con diagnóstico de Carcinoma de cuello uterino se concluyó que el estadío "0" representa $51.96 \%$ del total.

RELACION DE Ca. CERVIX IN SITU FRENTE A OTROS ESTADOS POR AÑOS

\begin{tabular}{|c|c|c|c|c|c|}
\hline \multirow[t]{2}{*}{ AÑO } & \multicolumn{2}{|c|}{ Ca, CERVIX CERO } & \multirow{2}{*}{$\begin{array}{l}\mathrm{Ca} . \\
\mathrm{N}^{0}\end{array}$} & \multirow{2}{*}{$\begin{array}{c}\text { CERVIX } \\
\begin{array}{c}1-I V \\
\end{array}\end{array}$} & \multirow{2}{*}{$\begin{array}{r}\text { TOTAL } \\
\mathrm{N}^{\prime}\end{array}$} \\
\hline & $\mathrm{N}:$ & $\%$ & & & \\
\hline 1970 & 18 & 43.90 & 23 & 56.10 & 41 \\
\hline 1971 & 15 & 40.54 & 22 & 59.46 & 37 \\
\hline 1972 & 11 & 37.93 & 18 & 62.07 & 29 \\
\hline 1973 & 20 & 62.50 & 12 & 37.50 & 32 \\
\hline 1974 & 18 & 48.65 & 19 & 51.35 & 37 \\
\hline 1975 & 25 & 65.79 & 13 & 34.21 & 38 \\
\hline 1976 & 12 & 80 & 3 & 20 & 15 \\
\hline TOTAL & 119 & 52 & 110 & 48 & 229 \\
\hline
\end{tabular}

CUADRO N: 3

Es conveniente anotar que para el último año del estudio -1976-, las pacientes con diagnóstico de estadío II a IV, se reritieron al centro de referencia para Cobaltorapia con sólo el informe de biopsila, en tanto que er los años precedentes, el Hospital realizó los estudios y análisis complemenarios previos al tratamiento con Cobalto.

En referencia a los datos demo- gráficos que se encontraron consignados en las 119 Historias Clínicas, encontramos como otros autores (3) (5) (6) (12) que entre los $30 \mathrm{y}$ 49 se encuentra la más alta incidencia, mostrando que el mayor riesgo corresponde a estos grupos de edad. Por debajo de los $20^{\prime}$ años, no se presentó ningún caso, la paciente de menor edad registró 21 años y la de mayor edad 63 años.

\begin{tabular}{lcc}
\hline & CARCINOMA IN SITU EDAD POR GRIIPOS & \\
\hline EDAD & No CASOS & $\%$ \\
\hline $15-19$ & 0 & 3.36 \\
$20-24$ & 4 & 5.88 \\
$30-34$ & 29 & 24.37 \\
$35-39$ & 23 & 19.33 \\
$45-49$ & 20 & 16.81 \\
$50-54$ & 8 & 6.72 \\
$55-$ más & 10 & 8.40 \\
\hline TOTAL & 119 & 100 \\
\hline
\end{tabular}


El $40.34 \%$ de lasł citologías fueron informadas como sospechosas
(III) y el $59.66 \%$ fueron informa das como positivas (IV ó V).

CITOLOGIA QUE MOTIVO LA BIOPSIA

\begin{tabular}{lcc}
\hline GRADOS & N: & $\%$ \\
III SOSPECHOSA & 48 & 40.34 \\
IV - V POSITIVA & 71 & 59.66 \\
\hline TOTALES & 119 & 100 \\
\hline
\end{tabular}

CUADRO $N: 5$

ESTUDIO ANATOMOPATOLOGICO

POST OPERACION

\begin{tabular}{lcccccc}
\hline INTERVENCION & \multicolumn{4}{c}{ D I A G N O S T I C O } \\
\hline & CERVICITIS & DISPLASIA & IN SITU & MOCROINV. & INVASIVO \\
\cline { 2 - 6 } \\
HISTERECTOMIA & $9(7.56 \%)$ & $3(2.52 \%)$ & $94(79 \%)$ & $4(3.36 \%)$ & $5(4.20 \%)$ \\
CONO & & & $3(2.52 \%)$ & $1(.84 \%)$ & \\
\hline
\end{tabular}

CUADRO N: 6

El cuadro $N^{\circ} 6$ muestra el diagnóstico Histológico de la pieza quirúrgica, observándose la coincidencia con el informe de Biopsia en el $81.52 \%$ de los casos con exactitud, correspondiendo a Carcinoma In situ.

Si tomamos por efecto de la conducta quirúrgica a seguir como correctos los diagnósticos de $\mathrm{Mi}$ croinvasivo y Displasia $-6.72 \%$, y asimilamos en el mismo sentido $7.56 \%$ de Cervicitis, llegamos a un gran total de $95.80 \%$ de seguridad en el diagnóstico, en referencia a la conducta seguida.
De los conos efectuados en uno, informado como Microinvasivo, se llevó la paciente a histerectomía.

La correlación Citología - Biopsia - Pieza Quirúrgica - Cuadro No 7, presenta la Citología sospechosa o positiva discriminada por años v/s la Patología de la Pieza Quirúr. gica, a partir del diagnósttico de Carcinoma In situ, establecido inicialmente por el informe Histológico de la Biopsia.

Haciendo el análisis del cuadro por años, podemos observar que sólo para el año 1975 se encuentran 
dos citologías falsas positivas, hecho éste, anotado por otros autores (6) (7) (10) (11) que creemos pudiera deberse a la extirpación total del Carcinoma In situ con la Bilopsia, no permitiendo corroborar el diagnóstico en la Pieza Quirúrgica.

Creemos que la Citología positiva (IV y V) es un buen indicio que, casi con seguridad, conduce al diagnóstico de Neoplasia, una vez se tome la Biopsia.

De todas maneras estamos convencidos que toda citología grado III o mayor es necesariamente tributaria de Biopsia de cuello uterino, siguiendo el esquema de manejo propuesto por Restrepo en nuestro Hospital (12), conducta que seguimos en todos estos casos.

\section{RESUMEN Y CONCLUSIONES}

Se analizan 119 Historias Clínicas correspondientes a pacientes con diagnósttico de $\mathrm{Ca}$. In situ de Cervix, del Servicio de Ginecología del Hospital de San Juan de Dios de Calii, las cuales se presentaron en el período: Enero $1^{\circ}$ de 1970 a Diciembre 31 de 1976.

La Biopsia de Cervix sirvió como punto de partida para hacer el estudio retrospectivo hacila la Citología $(40 \%$ sospechosa $60 \%$ positiva) que la motivó y el prospectivo hacia el diagnóstico Histopatológico de la pieza quirúrgica (Histerectomía o Cono).

La correlación resultante: CITOLOGIA, BIOPSIA, PIEZA QUIRURGICA lleva a la conclusión que se puede omitir el Cono de ru- tiina, en el manejo del $\mathrm{Ca}$. In situ de Cervix. Este procedimiento es la norma que se sigue en el Hospital de San Juan de Dios de Cali. El margen de error encontrado $4.2 \%$ establece un buen índice de seguridad para el tratamiento quirúrgico de estos casos en población hospitalaria, contribuyendo a disminuir los costos que presuponen efectuar Conización de Cervix en forma obligadia.

El Cono se reserva para tratamiento definitivo en aquellas pacientes que rechazan la extirpación de útero en favor de conservar su función obstétrica.

Los casos que resultaron con diagnóstico definilitivo de Carcinoma invasor, al seguir la secuencia Citología - Biopsia - Histerectomía, fueron referidos para Cobaltoterapia.

Los autores, de acuerdo a su experiencia, recomiendan la prueba de Schiller como un método apropiado y confiable para tomar la Biopsia de Cervix, pero no desconocen la seguridad que brinda la Colposcopia dirigida, utilizada en aquellos centros que poseen equipo y personal entrenado para su correcto uso.

\section{RESUMEN Y CONCLUSIONES}

119 Casos de $\mathrm{Ca}$ in situ de cérvix se analizan en la correlación citología - biopsia - pieza quirúrgica, concluyendo, aquí los autores, que se puede omitir la conización de rutina, ya que un margen de error de $4,2 \%$ parece un buen índice de seguridad. 
Ca In Situ de Cervix

Hospital de San Juan de Dios de Cali

$1.970-1.976$

\section{Correlación Citología Biopsia Pieza quirúrgica}

\begin{tabular}{|c|c|c|c|c|c|c|c|c|}
\hline \multirow[b]{2}{*}{ AÑo } & \multirow[t]{2}{*}{$\begin{array}{l}\text { No. Casos } \\
\text { Biopsia } \\
\text { Ca. In Situ }\end{array}$} & \multicolumn{2}{|c|}{ Citología } & \multicolumn{5}{|c|}{ Patología pieza quirúrgico y Citología } \\
\hline & & Sospechosa & Positiva & Cervicitis & Displasia & Micro & Invasivo & In SITU \\
\hline 1970 & 18 & 5 & 13 & $\frac{\text { III }}{\text { I }}$ & & & & 17 \\
\hline 1971 & 15 & 8 & 7 & III & & $\frac{\bar{V}}{I}$ & IV & 12 \\
\hline 1972 & 11 & 5 & 6 & & & $\frac{\bar{V}}{2}$ & & 9 \\
\hline 1973 & 20 & 10 & 10 & & & & $\frac{\text { IV }}{1}$ & 19 \\
\hline 1974 & 18 & 6 & 12 & $\frac{\text { III }}{\text { I }}$ & & & $\frac{\text { IVZ }}{2}$ & 15 \\
\hline 1975 & 25 & 8 & 17 & $\frac{\text { IIIIV IV }}{3}$ & $\frac{\text { III }}{\text { I }}$ & $\frac{\text { IVV }}{2}$ & $\frac{\text { IV }}{1}$ & 18 \\
\hline \multirow[t]{2}{*}{1976} & 12 & 6 & 6 & $\frac{\text { III }}{3}$ & $\frac{\text { IIII }}{2}$ & & & 7 \\
\hline & 119 & & & 9 & 3 & 5 & 5 & 97 \\
\hline
\end{tabular}


Admiten la conización como tratamiento definitivo en pacientes ansiosas de maternidad.

Cuando el diagnóstico definitivo fue carcinoma invasor se aconsejó cobaltoterapia.

El test de Schiller fue apropiado y confiable para dirigir la biopsia si blien se reconoce la seguridad de la colposcopia en esta selección.

\section{Summary and conclusions.}

119 cervix ca-insitu cases are analized on the correlation citology -biopsia- and surgical piece. The authours arrived to the conclusion that the rutine conization can be ommitted since 4.2 of error seems to be a good safety index.

They accept conization as a definitive treatment on patients who are anxious of maternity. When the final diagnosis was invading carcinoma, cobaltotherapy was advised.

The test of Schiller was apropiate and trustfull to direct the biopsia if the safety of the colcoscopy in selection is acknowledged.

\section{BIBLIOGRAFIA}

1. Amorocho, N., Henao, F., Carcinoma de Cervix en el Hospital de San Juan de Dios de Cali. XI Congreso Colombiano de Obst, y Ginec. Barranquilla 1975.

2. Creasman, W., Rutleedge, F.: Carcinoma in situ of the Cervix Obstet. Gynecol. 39: 375, 1972.

3. Gaitán Yanguas, M., Jordán, G.: Bo- letín del Insttituto Nal. de Cancerología Vol. 2, N.: 6. Bogotá, Colombia, 1974.

4. Griffiths, C., Younge, P.: Punch biopsy of the Cervix. Am. J. Obst. Gynec. 88, 1974.

5. Guzmán, N., Bueno, M.: Tendencia del cáncer de Cervix uterino en Cali. X Congreso Colombiano de Obst. y Gi. nec. Cali, 1973.

6. Jordán, G. y Cols.: Avances de el manejo de las neoplasias cervicales intra epiteliales. X Congreso Colombiano de Obst. y Ginec. Cali, 1973.

7. Scott, R.: Accurate Cervical Diagnostic studies. A necessity for individualized treatment of cancer of the uterine Cervix Obstet. Gynecol. Survey 24: 985, 1969.

8. Selim, M., So-bosita, J., Blair, O., Little, B.: Cervical Biopsy Versus Conization. Obst. and Gynec. and Gynec. 41, 1973.

9. Silbar, E., Woodruff, J.: Evaluation of biopsy, cone and hysterectomy seguence in intraepithelial carcinoma of the cervix, Obstet. Gynecol. 27: 89, 1966.

10. Spgut, H., Fechner, R.: Diagnóstico citológico de la displasia y carcinoma in situ del cuello uterino. Clin. Obst. y Ginec. Edit. interamericana, Diciembre 1967.

11. Stafl, A., Friedrich, Jr. E., Mattingly, R.: Diagnóstico de neoplasia cervical - reducción del riesgo de error. Clin. obst. y Ginec. Edit. Interamericana, Junio 1973.

12. Restrepo, H.: Correlación Cito - His tológica en Displasia y $\mathrm{Ca}$. in situ. $\mathrm{X}$ Congreso Colombiano de Obst. y Gi nec. Cali, 1973. 